\title{
Formaldehído: revisión bibliográfica sobre biomarcadores de efecto para la medición de la exposición ocupacional
}

\author{
Formaldehyde: A literature review on biomarkers of effect for measuring \\ occupational exposure
}

\section{Formaldeído: revisão da bibliografia sobre biomarcadores de efeito para medir a exposição ocupacional}

\author{
Iván Rodrigo Astros-Fonseca'; David Andrés Combariza-Bayona². \\ 1 Ingeniero químico. Especialista en Seguridad y Salud en el Trabajo, Facultad de Medicina, Universidad Nacional de Colombia. \\ iastrosf@unal.edu.co; ORCID: https://orcid.org/0000-0001-5211-1210. \\ 2 Medico, especialista en Medicina del Trabajo y Epidemiología, magíster en Toxicología, Facultad de Medicina, Universidad Nacional \\ de Colombia. dacombarizab@unal.edu.co; ORCID: https://orcid.org/0000-0002-2190-459X.
}

Recibido: 08/06/2018. Aprobado: 11/06/2019. Publicado: 01/10/2019

Astros-Fonseca IR, Combariza Bayona DA. Formaldehído: revisión de biomarcadores de efecto para la medición de la exposición ocupacional. Rev. Fac. Nac. Salud Pública. 2019;37(3):74-85. Dor:10.17533/udea.rfnsp.v37n3a09

\section{Resumen}

A pesar de las grades cantidades de formaldehído que se usa en la industria, la vigilancia de los efectos sobre la salud, derivados de la exposición ocupacional, sigue siendo un aspecto por mejorar en los programas de prevención de riesgos laborales. Objetivo: Identificar los diferentes biomarcadores de efecto que han sido propuestos para ser utilizados como indicadores indirectos de exposición a formaldehído y que se encuentran reportados en la literatura científica. Metodología: Se hizo una revisión bibliográfica desde el año 1990 hasta el primer trimestre del año 2018, usando los descriptores: "Formaldehído", "Biomarcador", "Exposición profesional". Se consultaron las bases de datos y se complementó la búsqueda inicial, mediante la consulta de la bibliografía citada por los autores de los artículos seleccionados. Se revisaron los resúmenes de los artículos y se seleccionaron aquellos que, luego de un análisis preliminar, se identificó contenían información relevante para la investigación. Resultados: Se encontraron 57 artículos relevantes para la revisión que contenían información sobre biomarcadores de efecto en poblaciones de trabajadores expuestos a formaldehído, algunos estudios en animales y células in vitro. Se resumieron los biomarcadores usados, listaron los estudios y describieron brevemente los hallazgos de cada uno. Conclusiones: La falta de conocimiento y entendimiento de los mecanismos toxicocinéticos y toxicodinámicos del formaldehído en los seres humanos limita la aplicación de un biomarcador de efecto que mida de manera práctica y sencilla la exposición al formaldehído exógeno.

-Palabras clave: formaldehído, biomarcadores, exposición ocupacional, efectos adversos, riesgos laborales, seguridad ocupacional, aductos de ADN, Colombia. 


\begin{abstract}
Despite the large amounts of formaldehyde used in the industry, monitoring the health effects of occupational exposure remains an aspect of further improvement in occupational risk prevention programs. Objective: To identify the different biomarkers of effect that have been proposed to be used as indirect indicators of formaldehyde exposure and that are reported in the scientific literature. Methodology: A bibliographic review of literature from 1990 to the first quarter of 2018 was made using the descriptors: "Formaldehyde", "Biomarker", "Professional exposure". Databases were consulted and the initial search was supplemented by consulting the bibliography cited by the authors of the selected articles. Abstracts of articles were reviewed and those that, after preliminary analysis, were determined to contain
\end{abstract}

information relevant to the research were selected. Results: The study found 57 articles relevant to the review which contained information on biomarkers of effect in populations of workers exposed to formaldehyde, some animal studies, and in vitro cells. Used biomarkers were summarized, studies were listed, and the findings of each of them were briefly described. Conclusions: The lack of knowledge and understanding of the toxicokinetic and toxicodynamic mechanisms of formaldehyde in humans limits the application of a biomarker of effect that measures in a practical and simple way exposure to exogenous formaldehyde. -Keywords: formaldehyde, biomarkers, occupational exposure, adverse effects, occupational hazards, occupational safety, DNA adducts, Colombia.

\section{Resumo}

Apesar da grande quantidade de formaldeído usado na indústria, o monitoramento dos efeitos na saúde, derivados da exposição ocupacional, continua sendo um aspecto a ser aprimorado nos programas de prevenção de riscos ocupacionais. Objetivo: Identificar os diferentes biomarcadores de efeito propostos para serem utilizados como indicadores indiretos da exposição ao formaldeído e relatados na bibliografia científica. Metodologia: Foi realizada uma revisão bibliográfica entre 1990 e o primeiro trimestre de 2018, utilizando os descritores: "Formaldeído", "Biomarcador", "Exposição Profissional". As bases de dados foram consultadas e a busca inicial foi complementada consultando a bibliografia citada pelos autores dos artigos selecionados. Os resumos dos artigos foram revisados e aqueles que, após análise preliminar, foram identificados, continham informações relevantes para a investigação. Resultados: Foram encontrados 57 artigos relevantes para a revisão que continham informações sobre biomarcadores de efeito em populações de trabalhadores expostos ao formaldeído, alguns estudos em animais e células in vitro. Os biomarcadores utilizados foram resumidos, os estudos listados e descritos brevemente os achados de cada um. Conclusões: A falta de conhecimento e entendimento dos mecanismos toxicocinéticos e toxicodinâmicos do formaldeído em humanos limita a aplicação de um biomarcador de efeito que mede de maneira prática e simples a exposição ao formaldeído exógeno.

------Palavras-chave: formaldeído, biomarcadores, exposição ocupacional, efeitos adversos, riscos ocupacionais, seguridade ocupacional, adutos de DNA, Colômbia.

\section{Introducción}

El formaldehído (FA) es una sustancia química del grupo de los aldehídos, que se obtiene por la oxidación catalítica del alcohol metílico. El nombre dado por la Unión Internacional de Química Pura y Aplicada (International Union of Pure and Applied Chemistry) es "metanal", pero también es conocido como "formalina", "aldehído fórmico", "óxido de metileno", "metanaldehído", "oxometano" o "formol" (disolución de FA en agua al $40 \%$ ).

El FA, cuya fórmula química es $\mathrm{CH}_{2} \mathrm{O}$, es una sustancia que se ha usado durante mucho tiempo y en muchas industrias a lo largo del mundo, debido a que es un producto que se obtiene a bajo costo y cuenta con más de cincuenta aplicaciones industriales conocidas, gracias a su capacidad de polimerización y formación de nuevas sustancias. Tiene un amplio espectro de aplicación, que va desde la industria de manufactura de resinas hasta los laboratorios de patología, cumpliendo funciones de preservante, desinfectante, producto intermedio de reacciones químicas y materia prima para producción de otros compuestos $[1,2]$.

Algunos usos que se le han dado son como líquido embalsamador de cadáveres o preservante de alimentos; en la industria textil y de cueros, como fijador de tintes y colorantes; y en la obtención de resinas, que son usadas en el tratamiento del papel, recubrimiento de superficies, espumas, aislamiento de madera, entre otros. Pero el mayor problema de su uso radica en el aprovechamiento de sus propiedades como insecticida, germicida y funguicida, lo que lo hace un componente importante de los detergentes y agentes de limpieza industrial y de equipos médicos, donde los trabajadores se exponen sin estar conscientes del riesgo que este representa [2-4]. 
Este amplio espectro de aplicación ha hecho que el FA sea un xenobiótico con gran impacto ambiental y en salud pública. En el año 2006, el FA fue clasificado por la Agencia Internacional para la Investigación del Cáncer (IARC) en el Grupo 1 (cancerígeno en humanos) [3], basándose en estudios epidemiológicos que cuentan con evidencia que indica un incremento en la incidencia de cáncer nasofaríngeo en trabajadores expuestos al FA, y en análisis que muestran una asociación positiva entre la exposición al FA y el desarrollo de leucemia y asma [2].

Aun así, la población en general no conoce los efectos sobre la salud que puede causar el FA y este sigue siendo usado sin las medidas de protección adecuadas. Incluso, en Colombia, se usa con poco control en algunas actividades de salud y en la industria de los cueros, funerarias y salones de belleza [5].

Los estudios en animales realizados por la IARC y otros investigadores han permitido entender algunos de los efectos y la forma en que actúa el FA en el organismo, indicando que este compuesto químico es absorbido rápidamente por el tracto gastrointestinal (ingestión) y por el sistema respiratorio (inhalación). Tiene una vida media de 1 a 1,5 minutos en el plasma sanguíneo [6-8], mientras que el ácido fórmico, derivado de su proceso de metabolismo, tiene una vida media en plasma sanguíneo de 55 minutos. Esto es importante señalarlo, porque los mayores daños causados por el FA se dan antes de ser metabolizado, y la porción de FA sin metabolizar que estará disponible en el organismo depende de la concentración a la que se ha expuesto la persona. Los efectos adversos agudos se presentan luego de 14 días de exposición, cuando las concentraciones son elevadas, sobrepasando los valores umbral límite (threshold limit value, TLV), mientras que los efectos adversos crónicos se manifiestan luego de 1 año de exposición continua [8].

A causa de la corta vida media del FA en el organismo, medirlo directamente en fluidos biológicos es un proceso complejo, ya que en la actualidad no se cuenta con una tecnología que establezca in situ la cantidad de FA en un tiempo menor al de su vida media [6-10]. Tampoco hay una forma indirecta de medirlo, debido a sus procesos en el organismo.

De esta manera, las restricciones tecnológicas y la corta vida media del FA han limitado el uso de biomarcadores de exposición, que miden de forma directa la cantidad del xenobiótico o su metabolito. Esta limitante ha obligado a los investigadores a buscar técnicas y métodos alternativos que relacionen la exposición al FA con cambios bioquímicos en el organismo; estos biomarcadores de efecto miden la respuesta del organismo a la exposición al xenobiótico [11].

El desarrollo de los biomarcadores de efecto ha obligado a los investigadores a entender en mayor medida el comportamiento del FA en el organismo y sus efectos sobre el mismo. El metabolito ácido fórmico es parcialmente incorporado a la ruta metabólica del carbono, y es oxidado a $\mathrm{CO}_{2}$ y $\mathrm{H}_{2} \mathrm{O}$, para ser eliminado vía respiratoria y renal $[8,10]$, por lo que cualquier resultado obtenido no permitirá estimar una cantidad representativa de FA dentro del organismo [3]. A nivel molecular, el FA reacciona con proteínas y el ADN, formando aductos con la hemoglobina y la albúmina. Además, es muy reactivo con aminas y amidas, formando puentes metilo con el ADN, lo cual ha llevado a los investigadores a explorar estos efectos como posibles indicadores de la exposición al FA [12-18].

Teniendo en cuenta lo anterior, se hizo una revisión de la literatura donde se buscó establecer los biomarcadores de efecto que han sido evaluados por los investigadores en función de los mecanismos de daño conocidos del FA y de los que se tiene registro hasta el año 2018.

\section{Metodología}

Para el desarrollo de la revisión y la localización de los documentos bibliográficos se consultaron las fuentes de documentos indexados. Se llevó a cabo una revisión bibliográfica desde el año 1990 hasta el primer trimestre del año 2018, usando los descriptores: "Formaldehído", "Biomarcador" y "Exposición profesional", en inglés y español.

Se consultaron las bases de datos PubMed, usando el descriptor "(("Formaldehyde"[Mesh]) AND "Occupational Exposure"[Mesh]) AND "Biomarkers" [Mesh]"; ScienceDirect, con el descriptor "Formaldehyde" AND "Occupational Exposure" AND "Biomarkers", y la herramienta OVID, que provee la Universidad Nacional de Colombia, usando el descriptor "Formaldehydelae, an, bl, im, me, pk, to, ur [Adverse Effects, Analysis, Blood, Immunology, Metabolism, Pharmacokinetics, Toxicity, Urine] AND Occupational Exposure/ae, an, sn [Adverse Effects, Analysis, Statistics \& Numerical Data] AND Biomarkers/an, bl, ge, im, me, pd, ur [Analysis, Blood, Genetics, Immunology, Metabolism, Pharmacology, Urine]".

Como complemento a la búsqueda inicial, se hizo una consulta de la bibliografía citada por los autores de los artículos seleccionados; se revisaron los resúmenes de los artículos y se seleccionaron aquellos que, luego de un análisis preliminar, se identificó contenían información relevante para la investigación, obteniendo así un total de 57 artículos que trataban el tema de biomarcadores de efecto por exposición a formaldehído.

Los descriptores usados corresponden a los conceptos principales del tema de investigación y buscan determinar si, en los trabajadores expuestos al FA, la exposición genera cambios biológicos que pueden ser medidos. 
Las palabras fueron validadas usando los descriptores "DeCS"y "MeSH". Luego de la búsqueda, se descargaron los documentos a los que se tenía acceso gratuito o usando el acceso de la Universidad Nacional de Colombia.

La búsqueda fue realizada por los autores del artículo. De igual modo, se extrajo la información relevante de los artículos. Se revisaron uno a uno y se resaltó la información relacionada con el mecanismo de daño, la forma de exposición, el efecto biológico medido y la conclusión del artículo. No se discriminó por técnica de análisis o la manera en que se tomó la muestra, dado que se buscaba explorar la medición de los efectos a la exposición.

El presente documento no requirió aval de comité de ética de la Universidad Nacional de Colombia, dado que es una revisión de información publicada en revistas indexadas y no se accede a información confidencial de pacientes o historias clínicas.

\section{Resultados}

Los resultados de la presente revisión son resumidos teniendo en cuenta la descripción realizada sobre los efectos tóxicos del fa en los seres humanos y los mecanismos descritos gracias a las investigaciones realizadas.

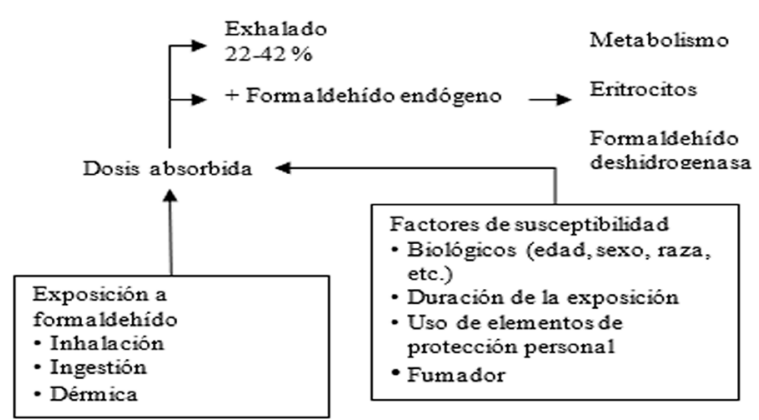

Figura 1. Toxicocinética del formaldehído

formando enlaces covalentes y complejos, principalmente puentes metilo. Estos permiten su posterior unión al glutatión, reducido para formar el hidroximetilglutatión, que a su vez sufre la acción de la formaldehído deshidrogenasa y origina el S-formilglutatión [2], sobre el cual actúa la S-formilglutatión hidrolasa, extrayendo la molécula de glutatión. Se produce de esta manera ácido fórmico, el cual es parcialmente incorporado a la ruta metabólica normal del carbono del organismo. El FA también es capaz de unirse a la albúmina sérica humana

\section{Toxicocinética y toxicodinamia}

Se ha determinado que existen tres vías de absorción del FA en los humanos: la primera y más común es por la vía respiratoria $[10,19,20]$, donde reacciona directamente con las mucosas y las células, afectando las proteínas y el ADN [2,21]. La segunda vía de exposición es por ingestión, y la tercera es vía dérmica [2].

La vía de exposición determina la cantidad de FA que será distribuido al plasma sanguíneo y los efectos agudos de la exposición al mismo. Por vía respiratoria e ingestión se ha determinado que genera inflamación, hiperplasia, cambios degenerativos, necrosis y metaplasia escamosa, mientras que la exposición dérmica ocasiona irritaciones e inflamaciones localizadas [5,22]. Estos efectos adversos no son significativos a la hora de determinar si un trabajador tiene una exposición crónica al FA, ya que los síntomas agudos de exposición varían entre las personas [8] y pueden ser confundidos con otras enfermedades, y los efectos crónicos se pueden presentar mucho tiempo después de la exposición y atribuírsele a otras causas [22]. La Figura 1 resumen la toxicocinética del formaldehído.

Las investigaciones han demostrado que el FA reacciona rápidamente con los grupos amino [23] y los grupos sulfuro [2] de las proteínas, y el ADN de las células,

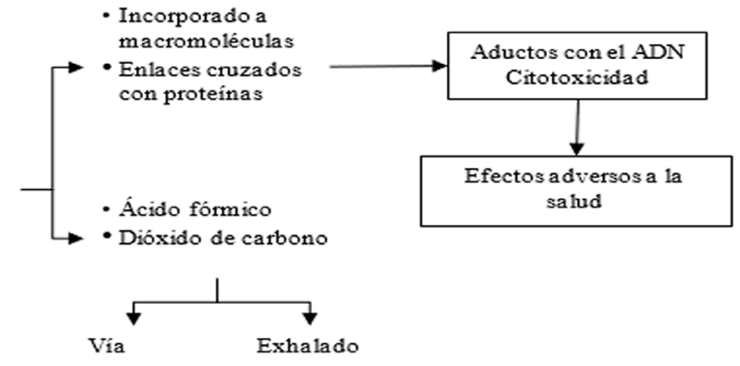

(human serum albumin, HSA), formando aductos en la misma $[3,12,23,24]$, los que pueden ser medidos mediante ensayos inmunológicos para detectar anticuerpos contra el complejo formaldehído-albúmina [12].

Aunque se conocen las reacciones que puede expresar el FA, la producción endógena de este compuesto, que hace parte de los ciclos metabólicos del organismo, presenta un inconveniente para determinar la cantidad de FA exógeno que ha ingresado al organismo y que puede causar daños. Además, se ha encontrado que los 
enlaces formados entre el FA y las proteínas, la albúmina o el ADN se ven alterados de forma positiva por la ingesta de aspirinas y el hábito de fumar, y los polimorfismos genéticos tienen una gran influencia sobre la formación de aductos, como consecuencia de diferencias en las enzimas que metabolizan el acetaldehído [12].

Como consecuencia de su clasificación en el grupo 1 de la IARC, la presencia del FA en ambientes laborales se ha regulado por la Conferencia Americana de Higienistas Industriales Gubernamentales (American Conference on Governmental Industrial Hygienists) hasta el umbral límite actual, reflejado en una concentración que no debe ser excedida en ningún momento durante la exposición laboral o la concentración instantánea a la cual nunca se debe exponer un trabajador durante su labor (threshold limit value celling, TLV-C) de 0,3 ppm $\left(0,370 \mathrm{mg} / \mathrm{m}^{3}\right)$ [1]. Los experimentos han determinado que a una exposición continua a una atmósfera, con una concentración de 1 ppm de FA $\left(1,23 \mathrm{mg} / \mathrm{m}^{3}\right)$, una tasa de respiración de $1 \mathrm{~m}^{3} / \mathrm{h}$ y un ritmo de bombeo cardíaco de 294 L / h, la máxima concentración de FA en sangre será de $0,0042 \mathrm{mg} / \mathrm{L}$ [12]. Pero esta concentración en sangre no está discriminada por FA endógeno o exógeno, lo cual limita la medición del mismo directamente en matrices biológicas, descartando el uso de un biomarcador de exposición para el monitoreo de la exposición al FA [14].

Y no es solo la limitante de medir directamente el FA; también se han hecho intentos de medir el ácido fórmico en sangre y orina, encontrando que no existen diferencias significativas de este metabolito entre personas expuestas y no expuestas a una concentración de 0,5 ppm de manera continua, la cual supera el TLV-C, haciendo que esto tampoco sea una forma práctica de monitoreo y control $[2,3]$.

\section{Biomarcadores de efecto}

Debido a que el FA es un aldehído reactivo, sus mecanismos de daño incluyen reacción con sitios nucleofílicos en moléculas, las que generan aductos con el ADN y las proteínas, los cuales pueden ser medidos mediante diferentes técnicas. La medición de estos cambios biológicos lleva al uso de biomarcadores de efecto, que miden indirectamente la exposición al FA en función de los cambios o daños que causa en el organismo. La Tabla 1 resume los productos de las reacciones entre el FA, las proteínas y el ADN que han sido identificados, así como la explicación del efecto y la matriz donde se han medido.

Tabla 1. Productos de reacción del formaldehído en el organismo

\begin{tabular}{|c|c|c|}
\hline Producto de reacción & Explicación & Matriz para medir \\
\hline Aductos N(6)-Lys199 & $\begin{array}{l}\text { La formación de aductos N(6)-Lys199 entre el FA con } \\
\text { la albúmina sérica humana (human serum albumin, } \\
\text { HSA) permite determinar la exposición por inhalación de } \\
\text { FA }[2,12,24,25]\end{array}$ & Sangre y saliva \\
\hline $\begin{array}{l}\text { Anticuerpos formaldehído- albúmina } \\
\text { sérica humana (antiFA-HSA) }\end{array}$ & $\begin{array}{l}\text { La formación de anticuerpos es un indicador de la } \\
\text { exposición, debido a los enlaces que forma el FA con la HSA, } \\
\text { lo cual genera una reacción alérgica [24] }\end{array}$ & Sangre \\
\hline $\begin{array}{l}\text { Interleucina - 4, 8, } 10 \\
(\mathrm{IL}-4,8,10)\end{array}$ & $\begin{array}{l}\text { El incremento de citoquinas es un marcador importante } \\
\text { de la respuesta inflamatoria a químicos irritantes como el } \\
\text { FA [19] }\end{array}$ & Suero \\
\hline $\begin{array}{l}\text { Isoprostanos } \\
(15-\text {-F2t-IsoP) }\end{array}$ & $\begin{array}{l}\text { Son compuestos bioactivos de tipo prostaglandina, } \\
\text { formados a partir de la peroxidación catalizada por } \\
\text { radicales libres de ácidos grasos esenciales. Son } \\
\text { marcadores de inflamación de las vías respiratorias }[26,27]\end{array}$ & Orina \\
\hline $\begin{array}{l}\mathrm{N}^{2} \text {-hidroximetildeoxiguanosina } \\
(\mathrm{HM}-\mathrm{dG})\end{array}$ & $\begin{array}{l}\text { Formación de aductos con el ADN y reticulaciones con } \\
\text { glutatión, haciendo que el ADN reaccione con los residuos } \\
\text { de lisina para producir } N^{6} \text {-formillisina, produciendo estrés } \\
\text { nitrosativo e inflamación oxidativa }[12,17,28]\end{array}$ & Sangre \\
\hline $\begin{array}{l}N^{6}-\text { hidroximetildeoxiadenosina } \\
\left(N^{6}-d A\right)\end{array}$ & $\begin{array}{l}\text { Formación de aductos con el ADN y reticulaciones con } \\
\text { glutatión, haciendo que el ADN reaccione con los residuos } \\
\text { de lisina para producir } N^{6} \text {-formillisina, y produciendo estrés } \\
\text { e inflamación oxidativa y nitrosativa [28] }\end{array}$ & Sangre \\
\hline N-metilvalina & $\begin{array}{l}\text { La reacción entre el FA y la hemoglobina para formar } \\
\text { N-metilenvalina, que luego es reducida a N-metilvalina, } \\
\text { permite diferenciar la cantidad total de FA que ha } \\
\text { reaccionado con las proteínas }[23,29]\end{array}$ & Sangre \\
\hline
\end{tabular}


Los cambios biológicos como consecuencia del daño oxidativo y nitrosativo al ADN son un factor importante en el momento de evaluar la exposición al FA, debido a que se ha observado la formación de formilfosfato, que tiene la capacidad de ligarse a proteínas y a la HSA [17], aunque no es un indicativo exacto de concentración o tiempo al que se ha estado expuesto.

También se han determinado otros cambios biológicos como consecuencia de la exposición al FA, entre ellos, el efecto inhibidor sobre la diferenciación de células madre en la médula ósea [30], razón por la cual se le ha relacionado con la leucemia [30-32]; efectos citogenéticos e inmunológicos [11], que se han vinculado a reducción de los linfocitos; mecanismos carcinogénicos como la inflamación, el estrés oxidativo y la apoptosis [25,33]; y monosomía del cromosoma 7 y trisomía del cromosoma 8 [30].

\section{Estudios de exposición ocupacional, in vitro y en modelos animales}

El conocimiento de los anteriores cambios biológicos ha llevado a los investigadores a realizar varios estudios en poblaciones expuestas al FA y en modelos animales. Se han encontrado cambios significativos al comparar trabajadores expuestos con no expuestos, y al evaluar animales o células in vitro expuestos a FA en condiciones de laboratorio. Las Tablas 2, 3 y 4 resumen algunos estudios al respecto y el biomarcador de efecto que se plantearon los investigadores.

Tabla 2. Resumen de biomarcadores utilizados en diferentes estudios en animales, para evaluar la exposición a formaldehído.

\begin{tabular}{|c|c|}
\hline Biomarcador de efecto & Resumen del estudio \\
\hline $\begin{array}{l}\text { Alteraciones de los } \\
\text { microácido ribonucleicos } \\
\text { (microARn) }\end{array}$ & $\begin{array}{l}\text { Se hizo un estudio en ratas y se midieron los cambios en los microARn de varios tejidos luego } \\
\text { de varios días de exposición; se encontró que las ratas expuestas presentaban alteraciones en } \\
\text { las células del epitelio nasal }[25,34] \text {. }\end{array}$ \\
\hline $\begin{array}{l}\text { Cambios en la } \\
\text { concentración de iones y } \\
\text { metabolitos presentes en la } \\
\text { orina de ratones }\end{array}$ & $\begin{array}{l}\text { Se estudió el cambio de iones y metabolitos en la orina de ratones expuestos a formaldehído } \\
\text { (FA), comparados con un grupo sin exposición. Por análisis estadístico se determinaron, como } \\
\text { candidatos a biomarcadores, el ácido hipúrico y la cinamoilglicina [35]. }\end{array}$ \\
\hline $\begin{array}{l}\text { Cambios histopatológicos } \\
\text { relacionados con } \\
\text { degeneración de células } \\
\text { tubulares del glomérulo de } \\
\text { ratas }\end{array}$ & $\begin{array}{l}\text { Los estudios histopatológicos revelaron que la exposición a altas dosis de FA se tradujo en } \\
\text { congestión glomerular, congestión focal y degeneración de las vacuolas de las células [6]. }\end{array}$ \\
\hline $\begin{array}{l}\text { Cambios en el micronúcleo } \\
\text { linfocitario (genotoxicidad) }\end{array}$ & $\begin{array}{l}\text { Se encontró que la exposición a FA de células eritropoyéticas de ratón induce en estas la } \\
\text { formación de micronúcleos [36] }\end{array}$ \\
\hline Conteo de linfocitos & $\begin{array}{l}\text { Estudios en animales han mostrado que la exposición al FA induce un incremento de } \\
\text { macrófagos y linfocitos [37] }\end{array}$ \\
\hline $\begin{array}{l}\text { Marcadores de inmunidad / } \\
\text { inflamación }\end{array}$ & $\begin{array}{l}\text { En ratas se encontró un incremento de las quimiocinas inflamatorias y cambios de la función } \\
\text { renal como consecuencia del incremento [38] }\end{array}$ \\
\hline $\begin{array}{l}\text { Medición de aductos con } \\
\text { N(6)-Lys199 }\end{array}$ & $\begin{array}{l}\text { Se midieron los aductos de N(6)-Lys199 en ratas expuestas, encontrando que los animales } \\
\text { expuestos al FA presentaban estos aductos, mientras que los controles no [17]. }\end{array}$ \\
\hline
\end{tabular}

Tabla 3. Resumen de biomarcadores utilizados en diferentes estudios en trabajadores, para evaluar la exposición a formaldehído.

\begin{tabular}{|c|c|}
\hline Biomarcador de efecto & Resumen del estudio \\
\hline $\begin{array}{l}\text { Biomarcadores de } \\
\text { genotoxicidad }\end{array}$ & $\begin{array}{l}\text { Se analizaron linfocitos en sangre y células de la mucosa bucal, y se encontraron diferencias } \\
\text { significativas }(p<0,001) \text { entre el número de micronúcleos, puentes nucleoplasmáticos y brotes } \\
\text { nucleares en las células de los trabajadores expuestos frente a los no expuestos. Otros estudios } \\
\text { han demostrado la formación de estos biomarcadores de genotoxicidad en otras poblaciones } \\
\text { de trabajadores expuestos a FA [18,39-42] }\end{array}$ \\
\hline $\begin{array}{l}\text { Biomarcadores de } \\
\text { inflamación }\end{array}$ & $\begin{array}{l}\text { Se estudió una población de trabajadores expuestos y se comparó con no expuestos. Se } \\
\text { midieron quimiocina }(C-X-C) \text { L11, timo y quimiocina regulada por activación, la proteína } C \\
\text { reactiva (CRP) y el ligante inductor de apoptosis relacionado con el factor de necrosis tumoral. Se } \\
\text { encontraron reducciones significativas de estos }(p<0,05) \text { en los trabajadores expuestos [33] }\end{array}$ \\
\hline $\begin{array}{l}\text { Cambios en el } \\
\text { micronúcleo linfocitario } \\
\text { (genotoxicidad) }\end{array}$ & $\begin{array}{l}\text { Se realizaron varios estudios a poblaciones de trabajadores expuestos y se observaron } \\
\text { diferencias significativas }(p<0,05) \text { con el grupo de control [13]. }\end{array}$ \\
\hline
\end{tabular}




\begin{tabular}{|c|c|}
\hline Biomarcador de efecto & Resumen del estudio \\
\hline $\begin{array}{l}\text { Conteo } \\
\text { de células sanguíneas }\end{array}$ & $\begin{array}{l}\text { Reducción de la cantidad de células T, CD8+ y células de memoria en los trabajadores } \\
\text { expuestos [33] }\end{array}$ \\
\hline Conteo de células blancas & $\begin{array}{l}\text { Se hizo un conteo de células blancas de los trabajadores expuestos y se comparó con no } \\
\text { expuestos, y se obtuvo una reducción significativa de células mieloides }(p=0,0016)[30] \text {. } \\
\text { Se expusieron células humanas a diferentes concentraciones de FA, encontrando que el FA } \\
\text { causa la muerte de las células, dependiendo de la concentración a la que sean expuestas } \\
(p<0,05)[43]\end{array}$ \\
\hline Conteo de linfocitos & $\begin{array}{l}\text { Mediante el análisis de un estudio transversal, se evaluaron los cambios en los linfocitos T } \\
\text { CD4+ y CD8+ de trabajadores expuestos (reducción de linfocitos del } 13 \% ; p<0,01) \text { [35]. } \\
\text { Se evaluó el número de linfocitos totales en trabajadores expuestos y se comparó con el control, } \\
\text { encontrando que el número total de células presentó una reducción significativa }(p=0,037) \text { en } \\
\text { los trabajadores expuestos [44]. } \\
\text { Se evaluó un grupo de trabajadores expuestos a FA }(n=85) \text { y se compararon los resultados con } \\
\text { un grupo de control }(n=87) \text {. Se midieron los porcentajes de linfocitos como biomarcadores de } \\
\text { inmutoxicidad, y se encontró que los trabajadores expuestos tenían alteraciones significativas en } \\
\text { los porcentajes de linfocitos Ty B [45]. }\end{array}$ \\
\hline
\end{tabular}

Desarrollo de anticuerpos

contra el complejo FA albúmina sérica humana (human serum albumin,

Se hizo el estudio sobre trabajadores expuestos a altas concentraciones de FA y se encontró que existe un cambio significativo en la respuesta inmunológica al complejo FA-HSA $(p=0,033)$ [24]

HSA)

Efectos de la exposición sobre la actividad de la acetilcolinesterasa y alcohol deshidrogenasa ॥I

En el estudio se determinó que hubo un incremento significativo en la actividad de la acetilcolinesterasa $(p<0,01)$ en los trabajadores expuestos, comparados con los no expuestos [46]
Frecuencias de monosomía, trisomía, tetrasomía y aberraciones estructurales de múltiples cromosomas

Incremento de citoquinas IL-6, IL-8, MCP-1

Marcadores de inmunidad / inflamación

Medición de ácido fórmico en orina, daño y frecuencia de daño citogénico
Se detectaron incrementos significativos $(p<0,05)$ de los cambios en cromosomas en los trabajadores expuestos a FA, comparados con los controles [47]

La comparación de células A549 cultivadas in vitro y expuestas a FA mostró un incremento de la producción de citoquinas respecto a las células control $(p<0,05)$ [19]

Se evaluaron 38 marcadores, de los cuales se obtuvieron diferencias significativas en 4 de ellos $(p<0,01)$, siendo menores para los trabajadores expuestos que para los no expuestos [33].

Se hizo el estudio en trabajadores de salones de belleza expuestos a productos con fa y se compararon contra trabajadores que no usaban estos productos. Se encontraron diferencias significativas $(p<0,001)$ en la cantidad de ácido fórmico en orina y el daño citogénico [48]

Medición de factores

de genotoxicidad

(aberraciones

cromosómicas,

intercambio de

cromatina, mutaciones

de hipoxantina-guanina-

fosforribosil-transferasa

(HPRT) y capacidad de

reparación del ADN)

Medición de momento de cola (Olive Tail Moment, Отм) y reticulaciones de proteína de ADN (DPC)

Se hizo un estudio en mujeres trabajadoras de laboratorio expuestas a FA y en un grupo de mujeres expuestas a FA y otros solventes; se compararon contra un grupo de control y se encontraron diferencias significativas ( $p=0,00354$ y 0,00104$)$ en la actividad apoptótica y las aberraciones cromosómicas en linfocitos, siendo mayores en las trabajadoras expuestas a FA [49]

Se realizaron medidas del OTM, DPC y micronúcleos en los linfocitos de trabajadores expuestos y no expuestos al FA, y se determinó que se tienen incrementos significativos de estos parámetros $(p<0,05)$ en los trabajadores expuestos, y que dichos valores eran dependientes de la dosis y el tiempo de exposición [16].

Se determinó que los cambios de отм son dependientes de la dosis y frecuencia de exposición al FA, siendo mayor en los trabajadores más expuestos $(p<0,01)$. Se correlacionaron las mediciones de daño al ADN y a los cromosomas, teniendo en cuenta los polimorfismos genéticos de los trabajadores, comparados con el grupo control [50] 


\begin{tabular}{|c|c|}
\hline Biomarcador de efecto & Resumen del estudio \\
\hline $\begin{array}{l}\text { Medición de reticulaciones } \\
\text { de las proteínas del ADN y } \\
\text { niveles de p53 }\end{array}$ & $\begin{array}{l}\text { Se midieron las reticulaciones de proteína de ADN y los niveles de p53 en trabajadores } \\
\text { expuestos y no expuestos, y se encontró un incremento de los dos factores en los trabajadores } \\
\text { expuestos [51] }\end{array}$ \\
\hline $\begin{array}{l}\text { Medición del compuesto } \\
15-\mathrm{F}_{2 t} \text {-IsoP tipo } \\
\text { prostaglandina }\end{array}$ & $\begin{array}{l}\text { Se midieron las concentraciones de } 15-\mathrm{F}_{2 \mathrm{t}} \text {-IsoP — - relacionado con el estrés oxidativo_- en la } \\
\text { orina de los trabajadores expuestos y no expuestos, y se encontró que los primeros presentan } \\
\text { niveles superiores [27] }\end{array}$ \\
\hline $\begin{array}{l}\text { Medición de aductos con } \\
\text { N(6)-Lys199 }\end{array}$ & $\begin{array}{l}\text { Se hallaron tres variaciones generadas por aductos a la N (6)-Lys199 del plasma en trabajadores } \\
\text { expuestos a FA [12] }\end{array}$ \\
\hline $\begin{array}{l}\text { Se midieron varios } \\
\text { biomarcadores } \\
\text { de genotoxicidad: } \\
\text { aberraciones } \\
\text { cromosómicas, daño en el } \\
\text { ADN, pruebas citogenéticas } \\
\text { (micronúcleos, MN; } \\
\text { intercambio de cromátidas } \\
\text { hermanas, scE), ensayo } \\
\text { de cometa y prueba de } \\
\text { micronúcleos con bloqueo } \\
\text { de citocinesis (CBMN) }\end{array}$ & $\begin{array}{l}\text { Se evaluó una población de trabajadores expuestos al fa y se determinó que estos presentaban } \\
\text { valores mayores de los biomarcadores de genotoxicidad que los trabajadores no expuestos, } \\
\text { luego de hacer un análisis estadístico teniendo en cuenta varios factores, como dieta, sexo, } \\
\text { edad, fumar, entre otros }[15,52,53]\end{array}$ \\
\hline
\end{tabular}

Tabla 4. Resumen de biomarcadores utilizados en diferentes estudios en células in vitro para evaluar la exposición a formaldehído.

\begin{tabular}{|c|c|}
\hline Biomarcador de efecto & Resumen del estudio \\
\hline $\begin{array}{l}\text { Aneuploidía del cromosoma } \\
7 \text { y } 8 \text { en células progenitoras } \\
\text { mieloides }\end{array}$ & $\begin{array}{l}\text { Se cultivaron las colonias que dan lugar a granulocitos y macrófagos a partir de células } \\
\text { mononucleares [36]. } \\
\text { Se cultivaron células hematopoyéticas de humanos, las cuales fueron expuestas a } \\
\text { FA, encontrando un incremento en la frecuencia de células con aneuploidía en los } \\
\text { cromosomas } 7 \text { y } 8 \text { [54] }\end{array}$ \\
\hline $\begin{array}{l}\text { Incremento de citoquinas IL-6, } \\
\text { IL-8, MCP-1 }\end{array}$ & $\begin{array}{l}\text { La comparación de células A549 cultivadas in vitro y expuestas a FA mostró un incremento } \\
\text { de la producción de citoquinas respecto a las células control }(p<0,05)[19]\end{array}$ \\
\hline $\begin{array}{l}\text { Medición de } \mathrm{N}^{2} \text { - } \\
\text { hidroximetildeoxiguanosina } \\
\text { (HM-dG) }\end{array}$ & $\begin{array}{l}\text { Debido a que el N²-HM-dG es un producto de la reacción del FA con la guanina, su } \\
\text { medición permite tener un biomarcador de prevalencia de las modificaciones del ADN por } \\
\text { efecto del FA [55]. } \\
\text { Considera que el producto de degradación N²-HM-dG es un excelente biomarcador, } \\
\text { por su relación con los aductos en el ADN y la modificación de proteínas causadas por el } \\
\text { FA [14] }\end{array}$ \\
\hline $\begin{array}{l}\text { Medición de } \mathrm{N}^{2}-\mathrm{HM}-\mathrm{dG} \text { y } \mathrm{N}^{6}- \\
\text { hidroximetildeoxiadenosina } \\
\left(\mathrm{N}^{6}-\mathrm{dA}\right)\end{array}$ & $\begin{array}{l}\text { Se miden los productos de la reacción del FA con la guanina y la adenina, y se encuentra } \\
\text { que en las células expuestas estos productos son mayores que en las no expuestas [28] }\end{array}$ \\
\hline $\begin{array}{l}\text { Número de células viables frente } \\
\text { a los datos de absorbancia }\end{array}$ & $\begin{array}{l}\text { Se realizó un ensayo in vitro con células epiteliales del pulmón A549 y se determinó que la } \\
\text { exposición al FA incrementa de forma significativa }(p<0,05) \text { la producción de citoquinas } \\
\text { en las células [19] }\end{array}$ \\
\hline
\end{tabular}

A pesar de tener varias propuestas para medir la exposición al FA, ninguna de estas es específica, y algunos de los biomarcadores medidos se pueden ver afectados por la presencia de otras sustancias químicas en el ambiente o por factores genéticos de los individuos expuestos $[32,34,46]$.

\section{Discusión}

Aunque los investigadores han logrado determinar una relación positiva entre los diferentes biomarcadores de efecto y la exposición al FA, no se tiene aún un método de monitoreo y control de la exposición en trabajadores que sea práctico, ya sea porque los métodos de 
determinación o de medición de los cambios biológicos por la exposición son complejos y costosos, o porque no se dilucida, en su totalidad, el mecanismo o la relación entre el biomarcador de efecto y la exposición a FA. En Colombia, el sistema de riesgos laborales no contempla el seguimiento médico por exposición a FA, y no se efectúa, de forma rutinaria, ninguno de los ensayos para medición de alguno de los biomarcadores presentados anteriormente, por lo cual es de vital importancia contar con investigaciones nacionales que desarrollen alguna de las metodologías para poder contar con estas herramientas de seguimiento a la salud de los trabajadores [56,57].

Los estudios realizados en las dos últimas décadas muestran que los cambios biológicos ocasionados por acción del FA generan daños al ADN y las proteínas de las células, en mayor cantidad en los sitios de contacto inmediato del xenobiótico con el organismo (células del epitelio nasal, la boca y la dermis), que en las células distantes, como son las de la medula ósea o tejido neuronal $[14,25,39,57,58]$.

El impacto en las células del epitelio nasal y la boca se debe a que la albúmina es abundante en los fluidos respiratorios y la saliva, lo cual incrementa el riesgo de daño por formación de aductos al ADN o con la albúmina. Debido a que el FA reacciona rápidamente en el sitio de contacto, la exposición aguda se relaciona más con molestias como irritación o tos, y los estudios han revelado que no se encuentran concentraciones representativas de FA o ácido fórmico en orina o sangre tras la exposición de los trabajadores [28].

Además, se ha determinado que los efectos adversos son diferentes entre las personas, como consecuencia de: polimorfismos de la alcohol deshidrogenasa, que es capaz de oxidar y detoxificar el FA [13]; la concentración en el aire, cuyo valor promedio ponderado en el tiempo (time weighted average, TWA) para una jornada de 8 horas de trabajo debe ser de 0,1 ppm [1]; y del tiempo de exposición, del género y de la edad de la persona [49]. Los hábitos de vida como fumar o ingerir alcohol también modifican los resultados que se pueden obtener de los análisis de los biomarcadores [46].

Debido a que el Fa puro es un gas incoloro irritante a temperatura y presión ambiente, siempre se ha comercializado en soluciones de agua y metanol. Todas estas soluciones corresponden a mezclas con 30 al $40 \%$ de FA y $15 \%$ de metanol, como agente estabilizante [59]. Se sabe que, a pesar de estar en solución acuosa, el FA se continúa comportando como agente reductor, con alta reacción con aminas y compuestos sulfurados, lo cual ha sido el punto de partida de las diferentes investigaciones para el desarrollo de biomarcadores de efecto, ya sea evaluando los daños al ADN o las reacciones del FA con otras sustancias endógenas.
Por lo dicho, se debe tener especial énfasis en continuar con la identificación de biomarcadores de efecto, pues a nivel ocupacional, los trabajadores muchas veces someten a calentamiento las soluciones de FA, provocando la evaporación de la solución $\mathrm{y}$, posteriormente, inhalándolo en forma de gas. Lo anterior se debe, en parte, al desconocimiento de los efectos de dichas soluciones, o a que muchos fabricantes de estas soluciones u otros productos no reportan el nombre de FA en sus etiquetas, y usan los sinónimos, generando confusión.

\section{Conclusiones}

Los efectos del FA se deben a su rápida absorción por los tejidos de las mucosas nasales y orales, y a su rápida metabolización a ácido fórmico, lo que no permite cuantificar o diferenciar el FA endógeno del exógeno. Esto dificulta el uso de biomarcadores de exposición, por lo cual se deben desarrollar biomarcadores de efecto que permitan tener un mayor entendimiento y posibilidad de monitoreo temprano de los efectos adversos sobre la salud de las poblaciones expuestas a esta sustancia química.

Está demostrado que las concentraciones de FA en sangre y orina no se incrementan tras la exposición de los trabajadores a atmósferas que superan los valores límite, y su metabolito, el ácido fórmico, no es un biomarcador específico de la dosis interna de FA, por lo que es de vital importancia entender los mecanismos de metabolización del FA para poder desarrollar un biomarcador de efecto, midiendo sus efectos asociados a irritación de piel o de mucosas.

Se debe continuar trabajando en entender aún más la toxicocinética y tóxicodinamia del FA, para identificar los mecanismos de daño exclusivos del FA exógeno. Ello brindará un biomarcador de efecto que sea práctico y sencillo de medir, para llevar a cabo un monitoreo correcto de las poblaciones de trabajadores expuestos y así evitar reacciones alérgicas, exacerbación de enfermedades respiratorias o el desarrollo de cáncer, y que permita también identificar, de forma temprana, cambios del equilibrio fisiológico como consecuencia de los mecanismos oxidantes del FA en los tejidos.

Las metodologías descritas para la identificación de biomarcadores requieren ser desarrolladas en Colombia, con el fin de ofrecer una herramienta de seguimiento médico que permita realizar controles periódicos a todos aquellos trabajadores expuestos al FA, ya que, enmarcados dentro de la normatividad nacional vigente referente a riesgos laborales, es de vital importancia el seguimiento a las personas expuestas a sustancias químicas cancerígenas, independiente de la dosis y tiempo de exposición. 


\section{Agradecimiento}

Se agradece al "Programa de Lectura y Escritura Académicas" de la Universidad Nacional de Colombia (Programa LEA en la UN), por su constante apoyo en el desarrollo del presente artículo.

\section{Financiación}

El presente trabajo no fue financiado por ninguna institución.

\section{Conflicto de interés}

Este trabajo no tiene conflicto de interés para ninguno de los autores.

\section{Declaración de responsabilidad}

Se declara que los puntos de vista expresados son responsabilidad de los autores.

\section{Referencias}

1. ToxLogic. Review of 2016 ACGIH Formaldehyde: TLV (R) Chemical Substances Draft Documentation, Notice of Intended Change. Gaithersburg; 2016. pp. 1-8.

2. International Agency for Research on Cancer (IARC). Chemical Agents and Related Occupations. Vol. 100 F. A Review of Human Carcinogens. IARC monographs on the evaluation of carcinogenic risks to humans. 2012

3. International Agency for Research on Cancer (IARC). Formaldehyde, 2-butoxyethanol and 1-tert-butoxypropan-2-ol [internet]. IARC Monogr Eval Carcinog Risks Hum. 2006 [citado 2018 sep. 3]; 88:1-390. Disponible en: https://monographs.iarc.fr/ wp-content/uploads/2018/06/mono88.pdf

4. Dietrich CJ, Richards IS, Bernard TE, et al. Human stress protein response to formaldehyde exposure. Exp. Toxicol. Pathol. [internet]. 1996 [citado 2018 mar. 18]; 48(6):518-9. DoI: http:// dx.doi.org/10.1016/S0940-2993(96)80071-6.

5. Casas J, Araque L, Herrera D. Caracterización de la exposición ocupacional a formaldehído en trabajadores del sector salud y educación en Colombia 2004-2013 [Tesis de Maestría] [internet]. Bogotá: Universidad del Rosario; 2015 [citado 2018 abr. 18]. Disponible en: http://repository.urosario.edu.co/ handle/10336/10667/.

6. Golalipour MJ, Azarhoush R, Ghafari S, et al. Can formaldehyde exposure induce histopathologic and morphometric changes on rat kidney? Int. J. Morphol. 2009;27(4):1195-200.

7. Mitkus RJ, Hess MA, Schwartz SL. Pharmacokinetic modeling as an approach to assessing the safety of residual formaldehyde in infant vaccines. Vaccine. 2013;31(25):2738-43.

8. Cardozo R, Peñalver C, Rivas B, et al. Características epidemiológicas y ocupacionales de trabajadores expuestos al formaldehído en centros asistenciales. Inf Médico [internet]. 2007 [citado 2017 feb. 7]; 9(7):365-73. Disponible en: http://ezproxy. unal.edu.co/login?url=http://search.ebscohost.com/login.aspx?di rect $=$ true \&db=a9h\&AN=26615157\&lang=es\&site=eds-live. De acceso restringido.

9. Kleinnijenhuis AJ, Staal YCM, Duistermaat E, et al. The determination of exogenous formaldehyde in blood of rats during and after inhalation exposure. Food Chem. Toxicol. [internet]. 2013 [citado 2018 mar. 18]; 52:105-12. DOI: http://dx.doi. org/10.1016/j.fct.2012.11.008.

10. Riess U, Tegtbur U, Fauck C, et al. Experimental setup and analytical methods for the non-invasive determination of volatile organic compounds, formaldehyde and $\mathrm{NO}_{x}$ in exhaled human breath. Anal. Chim. Acta [internet]. 2010 [citado 2018 mar. 18]; 669(1-2):53-62. DoI: http://dx.doi.org/10.1016/j.aca.2010.04.049.

11. Gil Hernández F. El papel de los biomarcadores en toxicología humana [internet]. Granada: Departamento de Medicina Legal, Facultad de Medicina de la Universidad de Granada; 2000. Disponible en: https://www.ugr.es/ fgil/ biomarcadoresrevtoxicol.pdf.

12. Regazzoni LG, Grigoryan H, Ji Z, et al. Using lysine adducts of human serum albumin to investigate the disposition of exogenous formaldehyde in human blood. Toxicol. Lett. 2017;268:26-35. Dor: http://dx.doi.org/10.1016/j.toxlet.2017.01.002.

13. Fenech M, Nersesyan A, Knasmueller S. A systematic review of the association between occupational exposure to formaldehyde and effects on chromosomal DNA damage measured using the cytokinesis-block micronucleus assay in lymphocytes. Mutat. Res. 2016;770:46-57. DoI: http://dx.doi.org/10.1016/j. mrrev.2016.04.005.

14. Yu R, Lai Y, Hartwell HJ, et al. Formation, accumulation, and hydrolysis of endogenous and exogenous formaldehyde-induced DNA damage. Toxicol. Sci. [internet]. 2015 [citado 2017 may. 14]; 146(1):170-82. DoI: http://dx.doi.org/10.1093/toxsci/kfv079.

15. Costa S, Carvalho S, Costa C, et al. Increased levels of chromosomal aberrations and DNA damage in a group of workers exposed to formaldehyde. Mutagenesis [internet]. 2015 [citado 2017 abr. 18]; 30(4):463-73. DOI: http://dx.doi.org/10.1093/ mutage/gev002.

16. Lin D, Guo Y, Yi J, et al. Occupational exposure to formaldehyde and genetic damage in the peripheral blood lymphocytes of plywood workers. J. Occup. Health. 2013;55(4):284-91.

17. Edrissi B, Taghizadeh $\mathrm{K}$, Moeller BC, et al. Dosimetry of $\mathrm{N}^{6}$ formyllysine adducts following $\left[{ }^{13} \mathrm{C}^{2} \mathrm{H}_{2}\right]$-formaldehyde exposures in rats. Chem. Res. Toxicol. 2013;26(10):1421-3. DoI: http:// dx.doi.org/10.1021/tx400320u.

18. Viegas S, Nunes C, Malta-Vacas J, et al. Genotoxic effects in occupational exposure to formaldehyde: A study in anatomy and pathology laboratories and formaldehyde-resins production. J. Occup. Med. Toxicol. [internet]. 2010 [citado 2017 may. 14]; 5(1):25-32. DoI: http://dx.doi.org/10.1186/1745-6673-5-25.

19. Persoz C, Achard S, Leleu C, et al. An in vitro model to evaluate the inflammatory response after gaseous formaldehyde exposure of lung epithelial cells. Toxicol Lett. 2010;195:99-105.

20. Malek FA, Möritz KU, Fanghänel J. A study on specific behavioral effects of formaldehyde in the rat. J. Exp. Anim. Sci. 2003;43(3):160-70.

21. Malek FA, Möritz K-U, Fanghänel J. Effects of a single inhalative exposure to formaldehyde on the open field behavior of mice. Int J Hyg Environ Health [internet]. 2004 [citado 2017 abr. 18]; 207:1518. Dor: http://dx.doi.org/10.1016/S0939-8600(03)80009-3.

22. Noisel N, Bouchard M, Carrier G. Evaluation of the health impact of lowering the formaldehyde occupational exposure limit for Quebec workers. Regul. Toxicol. Pharmacol. 2007;48(2):118-27. DOI: http://dx.doi.org/10.1016/j.yrtph.2007.02.001. 
23. Bono R, Romanazzi V, Pirro V, et al. Formaldehyde and tobacco smoke as alkylating agents: The formation of $\mathrm{N}$-methylenvaline in pathologists and in plastic laminate workers. Sci. Total Environ. [internet]. 2012 [citado 2018 mar. 18]; 414:701-7. DoI: http:// dx.doi.org/10.1016/j.scitotenv.2011.10.047.

24. Pala M, Ugolini D, Ceppi M, et al. Occupational exposure to formaldehyde and biological monitoring of Research Institute workers. Cancer Detect. Prev. 2008;32(2):121-6. DoI: http:// dx.doi.org/10.1016/j.cdp.2008.05.003.

25. Rager JE, Moeller BC, Miller SK, et al. Formaldehyde-associated changes in micrornas: Tissue and temporal specificity in the rat nose, white blood cells, and bone marrow. Toxicol. Sci. 2014;138(1):36-46. DoI: http://dx.doi.org/10.1093/toxsci/kft267.

26. Amiri A, Turner-Henson A. The roles of formaldehyde exposure and oxidative stress in fetal growth in the second trimester. J. Obstet. Gynecol. Neonatal. Nurs. [internet]. 2017 [citado 2018 mar. 18]; 46(1):51-62. DoI: http://dx.doi.org/10.1016/j. jogn.2016.08.007.

27. Romanazzi V, Pirro V, Bellisario V, et al. $15-\mathrm{F}_{2 \mathrm{t}}$ isoprostane as biomarker of oxidative stress induced by tobacco smoke and occupational exposure to formaldehyde in workers of plastic laminates. Sci Total Environ [internet]. 2013;442:20-25. DOI: http://dx.doi.org/10.1016/j.scitotenv.2012.10.057.

28. Zhong W, Que Hee SS. Formaldehyde-induced DNA adducts as biomarkers of in vitro human nasal epithelial cell exposure to formaldehyde. Mutat. Res. 2004;563(1):13-24. DoI: http://dx.doi. org/10.1016/j.mrgentox.2004.05.012.

29. Bono R, Vincenti M, Schiliro' T, et al. N-Methylenvaline in a group of subjects occupationally exposed to formaldehyde. Toxicol. Lett. 2006;161(1):10-17. DoI: http://dx.doi.org/10.1016/j. toxlet.2005.07.016.

30. Zhang L, Tang X, Rothman $\mathrm{N}$, et al. Occupational exposure to formaldehyde, hematotoxicity, and leukemia-specific chromosome changes in cultured myeloid progenitor cells. Cancer Epidemiol. Biomarkers Prev. 2010;19(1):80-88. DoI: http://dx.doi. org/10.1158/1055-9965.EPI-09-0762.

31. Zhang L, Steinmaus C, Eastmond DA, et al. Formaldehyde exposure and leukemia: A new meta-analysis and potential mechanisms. Mutat. Res. 2009;681(2-3):150-68. DoI: http:// dx.doi.org/10.1016/j.mrrev.2008.07.002.

32. Wei C, Chen M, You H, et al. Formaldehyde and co-exposure with benzene induce compensation of bone marrow and hematopoietic stem/progenitor cells in BALB/c mice during post-exposure period. Toxicol Appl Pharmacol [internet]. 2017 [citado 2018 mar. 18]; 324:36-44. DoI: http://dx.doi.org/10.1016/j.taap.2017.03.024.

33. Seow WJ, Zhang L, Vermeulen R, et al. Circulating immune/ inflammation markers in Chinese workers occupationally exposed to formaldehyde. Carcinogenesis. 2015;36(8):852-7. Dor: http:// dx.doi.org/10.1093/carcin/bgv055.

34. Wen H, Yuan L, Wei C, et al. Effects of combined exposure to formaldehyde and benzene on immune cells in the blood and spleen in BALB/c mice. Environ. Toxicol. Pharmacol. [internet] 2016 [citado 2018 mar. 18]; 45:265-73. DoI: http://dx.doi. org/10.1016/j.etap.2016.05.007.

35. Zhang J, Sun R, Chen Y, et al. Small molecule metabolite biomarker candidates in urine from mice exposed to formaldehyde. Int J Mol Sci. 2014;15(9):16458-68. DoI: https://doi.org/10.3390/ ijms150916458.

36. Bassig BA, Zhang L, Vermeulen $\mathrm{R}$, et al. Comparison of hematological alterations and markers of B-cell activation in workers exposed to benzene, formaldehyde and trichloroethylene.
Carcinogenesis. 2016;37(7):692-700. DOI: http://dx.doi. org/10.1093/carcin/bgw053.

37. Murta GL, Campos KKD, Bandeira ACB, et al. Oxidative effects on lung inflammatory response in rats exposed to different concentrations of formaldehyde. Environ. Pollut. 2016;211:20613. DOI: http://dx.doi.org/10.1016/j.envpol.2015.12.054.

38. Ramos C de O, Nardeli CR, Campos KKD, et al. The exposure to formaldehyde causes renal dysfunction, inflammation and redox imbalance in rats. Exp Toxicol Pathol [internet]. 2017 [citado 2018 mar. 18]; 69(6):367-72. DOI: http://dx.doi.org/10.1016/j. etp.2017.02.008.

39. Ladeira C, Viegas S, Carolino E, et al. Genotoxicity biomarkers in occupational exposure to formaldehyde--The case of histopathology laboratories. Mutat. Res. [internet]. 2011 [citado 2018 mar. 18]; 721(1):15-20. DoI: http://dx.doi.org/10.1016/j. mrgentox.2010.11.015

40. Costa S, Costa C, Silva S, et al. Cytogenetic alterations in formaldehyde exposed workers measured in a target and distal tissue. Toxicol. Lett. [internet]. 2013;221(2013):S63. Disponible en: https:/www.sciencedirect.com/science/article/ pii/S0378427413002294?via\%3Dihub, DoI: http://dx.doi. org/10.1016/j.toxlet.2013.05.029.

41. Ladeira C, Gomes MC, Brito M. 75 XRCC3 Thr241Met polymorphism influence on genotoxicity biomarkers frequency in workers occupationally exposed to formaldehyde. Eur. J. Cancer [internet]. 2010 [citado 2018 mar. 18]; Supl. 8(5):20. Disponible en: http://www.embase.com/search/results?subactio $\mathrm{n}=$ viewrecord \& from=export\&id=L70192583\%5Cnhttp://dx.doi. org/10.1016/S1359-6349(10)70884-3\%5Cnhttp://sfx.library. uu.nl/utrecht?sid=EMBASE\&issn=13596349\&id=doi:10.1016/ S1359-6349(10)70884-3\&atitle=XRCC3+Thr241Met+. Acceso restringido.

42. Rivera C, Rosales J. Genotoxic damage and occupational exposure to formaldehyde in anatomic pathology laboratory workers. Toxicol. Lett. [internet]. 2015 [citado 2018 mar. 18]; 238(2, Supl.):S103. DoI: https://doi.org/10.1016/j.toxlet.2015.08.339.

43. Li Q, Mei Q, Huyan $\mathrm{T}$, et al. Effects of formaldehyde exposure on human NK cells in vitro. Environ Toxicol Pharmacol. 2013;36(3):948-55. DOI: https://doi.org/10.1016/j. etap.2013.08.005.

44. Hosgood III HD, Zhang L, Tang X, et al. Occupational exposure to formaldehyde and alterations in lymphocyte subsets. Am. J. Ind. Med. 2013;56(2):252-7. Dor: https://doi.org/10.1002/ajim.22088.

45. Costa S, Costa C, García-Léston J, et al. Human exposure to formaldehyde, a risk evaluation of occupational health effects. Toxicol Lett [internet]. 2014 [citado 2018 mar. 18]; 229:S116. Disponible en: http://www.embase.com/search/results?subactio $\mathrm{n}=$ viewrecord\&from=export\&id=L71631238\%5Cnhttp://dx.doi. org/10.1016/j.toxlet.2014.06.419\%5Cnhttp://sfx.library.uu.nl/utr echt? sid=EMBASE\&issn=03784274\&id=doi:10.1016/j.toxlet.20 14.06.419\&atitle $=$ Human + exposure + to + . Acceso restringido.

46. Zendehdel R, Fazli Z, Mazinani M. Neurotoxicity effect of formaldehyde on occupational exposure and influence of individual susceptibility to some metabolism parameters. Environ Monit Assess. 2016; 188(1):648. DoI: http://dx.doi.org/10.1007/ s10661-016-5662-z.

47. Lan Q, Smith MT, Tang X, et al. Chromosome-wide aneuploidy study of cultured circulating myeloid progenitor cells from workers occupationally exposed to formaldehyde. Carcinogenesis [internet]. 2015 [citado 2017 may. 14]; 36(1):160-7. DoI: http:// dx.doi.org/10.1093/carcin/bgu229. 
48. Peteffi GP, Antunes MV, Carrer C, et al. Environmental and biological monitoring of occupational formaldehyde exposure resulting from the use of products for hair straightening. Environ. Sci. Pollut. Res. 2016;23(1):908-17. DoI: http://dx.doi. org/10.1007/s11356-015-5343-4.

49. Jakab MG, Klupp T, Besenyei K, et al. Formaldehyde-induced chromosomal aberrations and apoptosis in peripheral blood lymphocytes of personnel working in pathology departments. Mutat. Res. 2010;698(1-2):11-17. DoI: http://dx.doi.org/10.1016/j. mrgentox.2010.02.015.

50. Jiang S, Yu L, Cheng J, et al. Genomic damages in peripheral blood lymphocytes and association with polymorphisms of three glutathione S-transferases in workers exposed to formaldehyde. Mutat. Res. [internet]. 2010 [citado 2018 mar. 18]; 695(1-2):9-15. DOI: http://dx.doi.org/10.1016/j.mrgentox.2009.09.011.

51. Shaham J, Bomstein Y, Gurvich R, et al. DNA-protein crosslinks and p53 protein expression in relation to occupational exposure to formaldehyde. Occup. Environ. Med. 2003;60(6):403-9. DoI: http://dx.doi.org/10.1136/oem.60.6.403.

52. Costa S, Coelho $\mathrm{P}$, Costa $\mathrm{C}$, et al. Genotoxic damage in pathology anatomy laboratory workers exposed to formaldehyde. Toxicology. 2008;252(1-3):40-8. Dor: http://dx.doi.org/10.1016/j. tox.2008.07.056

53. Ladeira C, Gomes MC, Brito M. 74 Genotoxicity biomarkers in occupational exposure to formaldehyde in pathology anatomy laboratories. Eur. J. Cancer. Suppl. [internet]. 2010;8(5):19. DOI: http://dx.doi.org/10.1016/S1359-6349(10)70883-1.

54. Ji Z, Li X, Fromowitz M, et al. Formaldehyde induces micronucle in mouse erythropoietic cells and suppresses the expansion of human erythroid progenitor cells. Toxicol. Lett. [internet].
2014 [citado 2018 mar. 18]; 224(2):233-9. DoI: http://dx.doi. org/10.1016/j.toxlet.2013.10.028.

55. Pontel LB, Rosado IV, Burgos-Barragan G, et al. Endogenous formaldehyde is a hematopoietic stem cell genotoxin and metabolic carcinogen. Mol. Cell. [internet]. 2015 [citado 2017 may. 14]; 60(1):177-88. DoI: http://dx.doi.org/10.1016/j. molcel.2015.08.020.

56. $\mathrm{Lu} \mathrm{J}, \mathrm{Miao} \mathrm{J}, \mathrm{Su} \mathrm{T}$, et al. Formaldehyde induces hyperphosphorylation and polymerization of Tau protein both in vitro and in vivo. Biochim. Biophys. Acta - Gen. Subj. [internet]. 2013;1830(8):4102-16. DOI: http://dx.doi.org/10.1016/j. bbagen.2013.04.028.

57. Luo FC, Zhou J, Lv T, et al. Induction of endoplasmic reticulum stress and the modulation of thioredoxin-1 in formaldehydeinduced neurotoxicity. Neurotoxicology. 2012;33(3):290-8. DOI: http://dx.doi.org/10.1016/j.neuro.2012.02.004.

58. Zeller J, Neuss S, Mueller JU, et al. Assessment of genotoxic effects and changes in gene expression in humans exposed to formaldehyde by inhalation under controlled conditions. Mutagenesis. 2011;26(4):555-61. DoI: http://dx.doi.org/10.1093/ mutage/ger016.

59. Ministerio de Ambiente Vivienda y Desarrollo Territorial de Colombia. Guías para manejo seguro y gestión ambiental de 25 sustancias químicas. Bogotá: Publicaciones MinAmbiente; 2003. 